\title{
The Infant Microbiome
}

Complex Interactions

Share this:

Sara Copeland, DNP, ARNP, IBCLC, RLC ${ }^{a}$

The beneficial flora inhabiting the intestinal tract of an infant is extremely important for health, both in the short and long term. The establishment and further development of a healthy gut microbiome is a complicated interaction of factors in the infant. There are many elements that influence the development of the gut flora that extend beyond birth method and type of feeding. Maternal antibiotic use during pregnancy and during delivery is a common practice and can decrease the diversity of friendly biota in the mother and therefore decrease the amount and variety introduced to the infant. Antibiotic use in the mother while breastfeeding also has implications for health and diversity of the infant's gut bacteria because varying amounts of antibiotic medications transfer into breast milk. The use of probiotics is a common complimentary therapy that is used for a wide variety of reasons and is frequently used during pregnancy and lactation.

Keywords: microbiome; breastfeeding; probiotics; prebiotics

The human microbiome has been found to be an important factor in both states of health and in the acquisition of many health problems throughout the life span. The human microbiome is a term that comprises all of the microorganisms that colonize the human body. The largest concentration of these microorganisms, predominantly bacteria, is found in the intestines. The establishment of this microbiome starts at birth, or as new evidence suggests, possibly before birth. Many factors can influence the strains and numbers of gut bacteria colonization in the newborn. Delivery type, the use of prenatal and/or antenatal antibiotics, feeding method, time of infant's first bath, separation of mother and infant, and the use of antibiotics in the early neonatal period can affect the development of a robust and diverse microbiome (Mueller, Bakacs, Combellick, Grigoryan, \& Dominguez-Bello, 2015).

It has been well established that infants born vaginally and who are breastfed have greater numbers of more diverse beneficial bacteria in their intestinal tract (Rutayisire, Huang, Liu, \& Tao, 2016). Infants who were born via cesarean section and/or are formula-fed have fewer strains of bacteria that are known to be beneficial and greater numbers of bacteria that are potentially pathogenic in their gut; this is especially true for premature infants (Mueller et al., 2015). There are many more factors that can complicate the development of the friendly flora that go beyond birth type and feeding method.

a.sara04@ufl.edu

\section{Influencing Factors}

\section{Birth}

Infants born via cesarean section are not exposed to the vaginal flora of the mother during the birth process. Vaginal flora have been found to be of high importance to the colonization of the infant's gut with beneficial bacteria, so much so that it has been recommended that infants born by cesarean section be exposed to maternal vaginal contents soon after birth (Mueller et al., 2015). Commonly found strains of bacteria that live on the skin are the predominant strains that colonize the gut of infants born by cesarean section. These include Staphylococcus, Corynebacterium, and Propionibacterium, whereas the colonization of vaginally delivered infants are Lactobacillus, Prevotella, Sneathia, and Bifidobacterium (Mueller et al., 2015).

In general, the diversity of the newborn's gut is quite low initially. Exposure to healthy vaginal flora and breast milk are the primary sources of the beneficial bacteria early in the child's life (Matamoros, Gras-Leguen, Le Vacon, Potel, \& de La Cochetiere, 2013; Mueller et al., 2015). Over the following weeks to months, the microbial environment increases in diversity, and by around 3 years of age, the intestinal microbiome begins to resemble that of an adult (Matamoros et al., 2013; Voreades, Kozil, \& Weir, 2014). Multiple studies have found that infants born by cesarean section have major differences in the diversity of their microbiota for at least the first several months of life (Azad et al., 2016). One study found that these differences persist for up to 2 years (Neu, 2015). 


\section{Feeding}

Feeding method is another major factor in the colonization of a healthy microbiome. Infants who are fed breast milk substitute (infant formula) are at greater risk for low gut bacterial diversity and increased numbers of potentially pathogenic microbiota, whereas those who are breastfed have more gut bacteria diversity and increased numbers of strains that are generally considered to be part of a healthy gut flora (Voreades et al., 2014). The importance of breast milk for the health of an infant cannot be understated. Infants born via cesarean section but who were breastfed were found to have minor variations in the types and diversity of their gut flora by age 4 months when compared to breastfed vaginally delivered infants (Neu, 2015).

Breast milk contains prebiotic oligosaccharides. Prebiotics are substances that are indigestible by the gut but act to increase the growth of beneficial bacteria within the gut. Human milk also contains bacteria from the genus Streptococcus and Staphylococcus, which are known to be some of the first organisms to colonize the gut, as well as Lactobacillus and Bifidobacterium (Matamoros et al., 2013). Lactobacillus and Bifidobacterium are thought to be major components of a healthy microbiome and are found in much greater numbers in infants who are breastfed and who were born vaginally (Matamoros et al., 2013).

\section{Antibiotic Treatment}

Antibiotic use has very important implications for the robustness and diversity of an infant's intestinal flora. Anti-infective medications are overprescribed in all areas of healthcare including obstetrics and gynecology, neonatology, and pediatrics. Local and systemic antibiotics are commonly used during pregnancy to treat minor infections in the mother. Antibiotic use is also standard during labor and/or with cesarean section for many mothers, further decreasing the mother's microbial diversity. It is estimated that greater than $40 \%$ of pregnant women are given antibiotics immediately before delivery (Martinez de Tejada, 2014).

Infants born to mothers who were on antibiotics have decreased phylogenetic diversity of the bacteria present in the gut; ironically, this has been found to increase the risk of neonatal sepsis (Matamoros et al., 2013). One recent study found that infants exposed to intrapartum antibiotics had a significant decrease in the diversity of intestinal microbiota for at least 3 months and in some cases up to 1 year after intrapartum antibiotic exposure (Azad et al., 2016). Phylogenetic diversity is a measure of biodiversity of microorganisms. Infants are also frequently given a 48-hour course of broad-spectrum antibiotics while being assessed for possible sepsis. These antibiotics are typically started soon after birth, immediately after blood samples are drawn, including blood cultures and a complete blood count. The antibiotics are typically stopped after 48 hours if the blood culture has remained negative for growth and the infant is in good health.

Antibiotic treatment negatively impacts the balance between beneficial and potentially harmful gut bacteria. Studies have found that this disruption in bacterial proportions is detectable for at least 1 month after the end of antibiotic therapy (Matamoros et al., 2013). Infants who have had antibiotic treatment in their first days of life are at a higher risk for late-onset sepsis and have increased risk factors for obesity, metabolic diseases, celiac disease, and other inflammatory diseases (Matamoros et al., 2013; Mueller et al., 2015; Patel, Konduru, Patra, Chandel, \& Panigrahi, 2015; Sanz, 2013).

Another area that antibiotics impact the infant is through breast milk. This interaction may be twofold for the infant because maternal antibiotic use decreases the friendly flora in the mother as well as varying amounts of medication transfer into breast milk. The transfer of antibiotics may be detrimental to the infant's intestinal microbiome; nutrient malabsorption and/or diarrhea are often a result of maternal antibiotic treatment (Mathew, 2004). It is well-known that breast milk is the best form of infant nutrition and that it is extremely important in the health of the infant to receive breast milk. However, it is important to remember that even when a medication is considered "safe" for use with lactation, there may be health consequences for the infant. There is an extremely complex interplay of the microbial environments of the mother and the infant as well as health implications that extend far beyond minor gastrointestinal symptoms in the infant.

Antibiotic use is well-known to not only treat targeted microorganisms for which they are prescribed but also reduce the numbers of beneficial bacteria throughout the body. Regarding the treatment of a lactating mother, the reduction in friendly flora extends to the infant as well. The unfortunate truth about antibiotic use is that they may be absolutely necessary to treat some infections, and although many may be lifesaving, all antibiotic use is detrimental to the health of the gut microbiota. This is exhibited by many of the side effects that are common to antibiotic use, such as diarrhea, secondary opportunistic infections, and 
diaper dermatitis, to name a few. The global aspect of the health consequences of antibiotic use for the individual is largely unknown (Mathew, 2004; Mshvildadze \& Neu, 2010).

\section{Preterm Delivery}

Preterm infants are at a particularly high risk for suboptimal microbial colonization. Gestational age has been found to be associated with decreased microbial diversity (Matamoros et al., 2013). Preterm infants are more delicate than their full-term counterparts and, depending on gestational age, may require intensive medical treatments. Preterm infants are commonly separated from their mothers, have delayed first feedings, are often born by cesarean section, and are very commonly treated with broad-spectrum antibiotics while being assessed for possible sepsis (Mshvildadze \& Neu, 2010). Preterm infants are environmentally exposed to potentially pathogenic bacteria in the neonatal intensive care unit (NICU). It is also likely that many preterm infants are colonized with harmful bacteria at birth because of maternal infection and/or prenatal antibiotic exposure (Madan, Farzan, Hibberd, \& Karagas, 2012).

Each of the earlier mentioned risk factors decreases the colonization of beneficial bacteria and increases the risk of harmful colonization. The bacteria that are found in greater numbers in the intestinal tracts of preterm infants versus full-term infants are Enterobacteriaceae, Klebsiella pneumoniae, and Clostridium difficile. These bacteria are associated with disease and put the infant at higher risk for morbidity and mortality (Matamoros et al., 2013). Necrotizing enterocolitis (NEC) is of particularly high concern because it is a potentially life-threatening infection of the gut; however, there are many unknown consequences beyond NEC that need to be considered. Premature infants are at a higher risk for developmental delays and morbidity than full-term babies because of the decreased gestational time. A suboptimum gut flora may also contribute to this (Patel et al., 2015).

\section{Probiotics in Pregnancy and Lactation}

The use of probiotics has increased in recent years and is becoming a very common recommendation by healthcare providers to aid in the treatment of or recovery from various health concerns. Many studies have shown promising health benefits of supplementing the gut with friendly bacteria. In general, probiotics are considered safe during pregnancy and lactation. However, there are still many unknown aspects of what extrinsically changing the microbial balance may result in for the infant. Traditionally, it was thought that probiotics were not likely to transfer into breast milk and that breast milk contained only prebiotics. More recent evidence has found that breast milk does contain strains of beneficial bacteria thought to have come from the mother's intestinal tract. Studies of mothers taking Bifidobacterium supplements during pregnancy and lactation found increases in the numbers of and phylogenetic diversity of Bifidobacteria in the infants' gut flora (Matamoros et al., 2013).

The mechanism of how the beneficial bacteria get into the breast milk is not well-understood. By changing the gut flora of the mother, the types and amount of bacteria in the breast milk may change, although this has been understudied to date. There is evidence to support the use of prebiotics in pregnant and lactating mothers. Prebiotics have been found to positively affect the anti-inflammatory effects of breast milk (Kubota et al., 2014). This may be an important mechanism in the reduction of atopic disease, as well as other inflammatory diseases, found in children who are breastfed. Studies of prebiotics and probiotic supplementation in infants have demonstrated some beneficial effects in reducing common infantile conditions, such as atopic dermatitis/eczema and a reduction in various infections, including diarrhea and NEC (Matamoros et al., 2013).

\section{Implications for Infant Health}

Fetal and early newborn life are extremely important times for the initial development of a healthy microbiome. An unhealthy microbiome is associated with various health problems, both short and long term, including autoimmune diseases, allergies, inflammatory lung diseases, metabolic syndrome, obesity, type 1 and 2 diabetes mellitus, irritable bowel syndrome, and Crohn's disease, among others (Matamoros et al., 2013; Neu, 2015; Sanz, 2011). Premature birth may also be associated with an unhealthy microbiome in the mother. Premature infants are at higher risk for NEC, chronic lung disease, brain white matter disease, developmental delays, and other inflammatory diseases that are associated an unhealthy intestinal microbiome (Neu, 2015).

Each person's microbiome is unique. These microbiome variations are thought to begin with the initial colonization and development of gut flora in infancy and continue throughout each individual's entire life (Matamoros et al., 2013). It is of paramount importance for the lifelong health of an individual to have a healthy intestinal microflora which starts at or before 
birth. Birthing, feeding, and early infant care practices should be as supportive as possible to nurture a healthy microbiome (Mshvildadze \& Neu, 2010; Sanz, 2011).

\section{Recommendations for Lactation Consultants and Maternal-Child Health Providers}

Increasing the health of the microbiome increases the health of the host organism. The mother and child will benefit from diverse and healthy gut flora (Sanz, 2011). The Western diet and lifestyle is not typically supportive of a healthy gut microflora; however, there are many simple recommendations that can be made to increase the health of the microbiome. Diets rich in plant-based foods, and low in processed foods, can shift the balance of intestinal bacteria toward a more healthy balance (Matamoros et al., 2013).

Increasing the beneficial bacteria in the gut reduces gut and systemic inflammation and increases the immune system's ability to fight infections of various kinds (Sanz, 2011). This is very important for pregnant women because inflammation and subclinical infectious processes may play a role in preterm delivery and the health of the fetus (Neu, 2015; Patel et al., 2015). Providing a diet rich in diverse plant-based options, when solid foods are introduced, is also essential for a healthy gut in the infant (Sanz, 2011). Increasing breastfeeding exclusivity is another way to increase bacterial diversity in the infant. There is a growing body of evidence that shows that even a single feeding of formula can change the microbiome of an infant. The long-term repercussions of this are not known at this time (Mueller et al., 2015).

Prebiotics and probiotics are considered safe for use during pregnancy and lactation. The health benefits and/or risks are not fully known at this point. However, many studies have shown promising results from supplementing the mother and/or the infant with preand probiotics. The use of pre- and probiotics may be particularly helpful in strengthening the gut microbiome following the use of antibiotic therapy. If a breastfeeding mother has taken antibiotics, both the mother's and the infant's friendly flora may have suffered and a pre-and/ or probiotic supplementation may be of benefit to them. Only high-quality probiotic supplements manufactured by reputable sources should be taken by infants and pregnant or lactating women (Matamoros et al., 2013).

\section{Conclusion}

The intestinal microbiome is extremely important for the health of an individual. Infancy is a particularly critical time in the development of a healthy microbial environment in the gut. The traditional thought is that the intestinal colonization of an infant begins to develop soon after birth. Newer evidence suggests that the amniotic sac may be less sterile than once believed and that the infant may have bacterial exposure before birth. Studies have found correlations between the prenatal presence of some strains of bacteria in the placenta, umbilical cord, and amniotic sac with risk of preterm delivery. Many factors influence the development of a healthy and diverse microbiome in the infant. Type of birth, feeding method, environmental factors, and medical treatments are all extremely influential in the establishment of an infant's gut bacteria. There are many factors that may be less obvious and yet are also extremely important.

\section{References}

Azad, M., Konya, T., Persaud, R., Guttman, D., Chari, R., Field, C., ... Kozyrskyj, A. L. (2016). Impact of maternal intrapartum antibiotics, method of birth and breastfeeding on gut microbiota during the first year of life: A prospective cohort study. British Journal of Obstetrics and Gynaecology, 123(6), 983-993. http://dx.doi.org/10.1111/1471-0528.13601

Kubota, T., Shimojo, N., Nonaka, K., Yamashita, M., Ohara, O., Igoshi, Y., ... Kohno, Y. (2014). Prebiotic consumption in pregnant and lactating women increases IL-27 expression in human milk. British Journal of Nutrition, 111, 625-632. http://dx.doi.org/10.1017/S0007114513003036

Madan, J., Farzan, S., Hibberd, P., \& Karagas, M. (2012). Normal neonatal microbiome variation in relation to environmental factors, infection and allergy. Current Opinion in Pediatrics, 24(6), 753-759. http://dx.doi.org/10.1097/ MOP.0b013e32835a1ac8

Martinez de Tejada, B. (2014). Antibiotic use and misuse during pregnancy and delivery: Benefits and risks. International Journal of Environmental Research and Public Health, 11(8), 7993-8009. http://dx.doi.org/10.3390/ijerph110807993

Matamoros, S., Gras-Leguen, C., Le Vacon, F., Potel, G., \& de La Cochetiere, M.-F. (2013). Development of intestinal microbiota in infants and its impact on health. Trends in Microbiology, 21(4), 167-173. http://dx.doi.org/10.1016/j.tim.2012.12.001

Mathew, J. (2004). Effect of maternal antibiotics on breast feeding infants. Postgraduate Medical Journal, 80, 196-200. http://dx.doi .org/10.1136/pgmj.2003.011973

Mshvildadze, M., \& Neu, J. (2010). The infant intestinal microbiome: Friend or foe? Early Human Development, 86(Suppl. 1), 67-71. http://dx.doi.org/10.1016/i.earlhumdev.2010.01.018

Mueller, N., Bakacs, E., Combellick, J., Grigoryan, Z., \& DominguezBello, M. (2015). The infant microbiome development: Mom matters. Trends in Molecular Medicine, 21(2), 109-117. http:// dx.doi.org/10.1016/i.molmed.2014.12.002

Neu, J. (2015). Developmental aspects of maternal-fetal, and infant gut microbiota and implications for long-term health. 
Maternal Health, Neonatology, and Perinatology, 1, 6. http://dx.doi .org/10.1186/s40748-015-0007-4

Patel, K., Konduru, K., Patra, A., Chandel, D., \& Panigrahi, P. (2015). Trends and determinants of gastric bacterial colonization of preterm neonates in a NICU setting. PLoS One, 10(7), e0114664. http://dx.doi.org/10.1371/journal.pone.0114664

Rutayisire, E., Huang, K., Liu, Y., \& Tao, F. (2016). The mode of delivery affects the diversity and colonization pattern of the gut microbiota during the first year of infants' life: A systematic review. BMC Gastroenterology, 16, 86. http://dx.doi.org/ $\underline{10.1186 / \mathrm{s} 12876-016-0498-0}$

Sanz, Y. (2011). Gut microbiota and probiotics in maternal and infant health. The American Journal of Clinical Nutrition, 94(Suppl. 6), 2000S-2005S.

Voreades, N., Kozil, A., \& Weir, T. (2014). Diet and the development of the human intestinal microbiome. Frontiers in Microbiology, 5, 494. http://dx.doi.org/10.3389/ fmicb.2014.00494

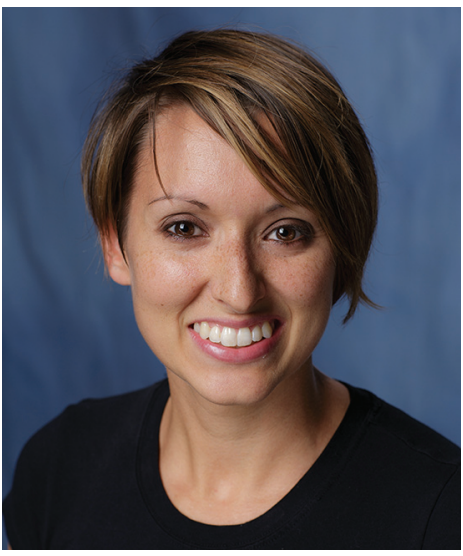

Sara has experience as a registered nurse $(\mathrm{RN})$ in the NICU, where she was involved in interdisciplinary developmental care and feeding committees aimed at bringing evidence-based practices in these areas to the unit. As an IBCLC, Sara has experience in the inpatient setting, primarily with preterm or compromised infants, and in the outpatient setting. Sara is currently a pediatric nurse practitioner specializing in breast feeding medicine and sees a wide variety of breastfeeding dyads and general pediatric patients in the outpatient setting.

\section{Your Guide to Breastfeeding Updated From Health and Human Services}

The U.S. Department of Health and Human Services Office on Women's Health (OWH) has updated their Your Guide to Breastfeeding publication, featuring information and support to help women breastfeed. Download and print a PDF version: https://www.womenshealth.gov/files/documents/ your-guide-to-breastfeeding.pdf Or, download it through the U.S. Government Bookstore, Apple's iBooks, Google Play, or Barnes and Noble. OWH is also offering the print version to health professionals and lactation consultants in bulk quantities (a minimum of 25 and a maximum of 500). To order hard copies, call the OWH Helpline at 1-800-994-9662.

Source: USBC 